\title{
A THREE-DIMENSIONAL OUTER MAGNETOSPHERIC GAP MODEL FOR GAMMA-RAY PULSARS: GEOMETRY, PAIR PRODUCTION, EMISSION MORPHOLOGIES, AND PHASE-RESOLVED SPECTRA
}

\author{
K. S. Cheng, ${ }^{1}$ M. Ruderman, ${ }^{2}$ AND L. ZhaNG ${ }^{1,3}$ \\ Received 1999 August 19; accepted 2000 February 17
}

\begin{abstract}
A three-dimensional pulsar magnetosphere model is used to study the geometry of outer magnetospheric gap accelerators, following seminal work of Romani and coworkers. The size of the outer gap is self-consistently limited by pair production from collisions of thermal photons from polar cap heating of backflow outer gap current with curvature photons emitted by gap-accelerated charged particles. In principle, there could be two topologically disconnected outer gaps. Conditions for local pair production such as local field line curvature, soft X-ray density, electric field, etc., support pair production inside an outer gap only between $r_{\text {in }}(\phi)$ (the radius of the null surface at azimuthal angle $\left.\phi\right)$ and $r_{\text {lim }}(\phi) \approx 6 r_{\text {in }}(\phi=$ $0) \ll R_{L}$ (the light cylinder radius). Secondary pairs, on the other hand, are produced almost everywhere outside the outer gap by collisions between curvature photons and synchrotron X-rays emitted by these secondary pairs. These processes produce a wide X-ray fan beam in the outgoing direction and a very narrow beam in the incoming direction for each outer gap. For pulsars with a large magnetic dipole inclination angle, part of the incoming $\gamma$-ray beam will be absorbed by the stellar magnetic field. If the surface magnetic field is dominated by a far off-center dipole moment (e.g., as in a proposed "plate tectonic" model), gravitational bending of photons from polar cap accelerators and their ultimate conversion into outflowing $e^{ \pm}$pairs can result in the quenching of one of these two outer gaps. Various emission morphologies for the pulsar (depending on magnetic inclination angle and viewing angle) are presented. Double-peak light curves with strong bridges are most common. From the three-dimensional structure of the outer gap and its local properties, we calculate phase-resolved spectra of gamma-ray pulsars and apply them to observed spectra of the Crab pulsar.
\end{abstract}

Subject headings: gamma rays: theory — pulsars: general — stars: magnetic fields

\section{INTRODUCTION}

"Outer gaps" are charge-deficient regions with a strong electric field along the magnetic field near the null charge surface $\boldsymbol{\Omega} \cdot \boldsymbol{B}$, where $\boldsymbol{\Omega}$ is the stellar angular velocity and $\boldsymbol{B}$ is the local magnetic field. It has been argued that such gaps should form in the outer magnetosphere regions of a pulsar (angular velocity $\boldsymbol{\Omega}$ and magnetic moment $\mu$ ) (Holloway 1973; Cheng, Ruderman, \& Sutherland 1976). Cheng, Ho, \& Ruderman (1986a, 1986b, hereafter CHR I and CHR II) (see also Ho 1989) considered the origin and structure of outer gaps and various general morphological features of their energetic radiation in a static magnetosphere approximation. CHR I suggested that four outer gaps in the $(\boldsymbol{\Omega}, \mu)$ plane can exist in the magnetosphere between the null surface and the light cylinder. From one pole, there are two outer gaps. One is long (labeled gap 1 in Fig. 1) and the other short (gap 3). Similarly, one long gap (gap 2) and one short gap (gap 4) exist associated with the opposite pole (see Fig. 1). The two outer gaps (i.e., 1 and 3, 2 and 4) associated with the same magnetic pole are actually one topologically connected outer gap; there are only two topologically disconnected outer gaps. CHR I assumed that the active region of the outer gap for radiation and pair production is restricted to the long gaps ( 1 and 2)-a slablike geometry with a small extension in the azimuthal direction. This assumption is valid only for large inclination angles $(\alpha)$. For a nearly aligned rotator, gaps $1,2,3$, and 4 should all be

\footnotetext{
${ }^{1}$ Postal address: Department of Physics, University of Hong Kong, Pokfulam Road, Hong Kong, China.

${ }^{2}$ Department of Physics, Columbia University. China.
}

active, although it is not easy to determine exactly at what inclination angle this transition will occur. If the distance $\left(r_{\text {in }}\right)$ to the null surface of gap $1(2)$ is much less than the light cylinder radius $\left(R_{L}\right)$, i.e., $r_{\text {in }} / R_{L} \sim 4 /\left(9 \tan ^{2} \alpha\right)<1$, which implies $\alpha>34^{\circ}$, gap 1 and gap 2 are likely the dominant ones. Inside the outer gap, electrons and positrons are accelerated to extreme relativistic energies in opposite directions and result in two crossed radiation fan beams. CHR I assumed that $\gamma$-ray emission occurs only close to the last closed field line in the plane of the dipole and rotation axes. Four identical fan beams (two toward and two away from the light cylinder) are emitted from the pulsar since the charged particles of positive and negative charges are oppositely accelerated in the outer gap. The morphological features of the emission in the CHR model are as follows: (1) the emission pattern is a double fan beam with the $\gamma$-ray emission produced tangential to the last closed magnetic field line; (2) the double-peaked $\gamma$-ray pulse profile consists of one beam from one outer gap and another beam (parallel to the former) from the opposite outer gap; two observed pulses with similar strength would be expected; and (3) the measured phase separation between the two peaks is determined by the time travel difference between these two outer gaps, relativistic aberration of emission, and the bending of the magnetic field lines near the light cylinder. The emission from each peak is highly cusped because of the relativistic aberration, so there will be some bridge emission but very little other off-pulse emission.

EGRET has observed the phase-resolved emission characteristics such as pulse profiles and phase-resolved spectra of the Crab, Vela, PSR B1706-44, and Geminga $\gamma$-ray pulsars, but PSR B1055-52 and PSR B1951+32 do not have sufficient photon counting statistics to perform a 


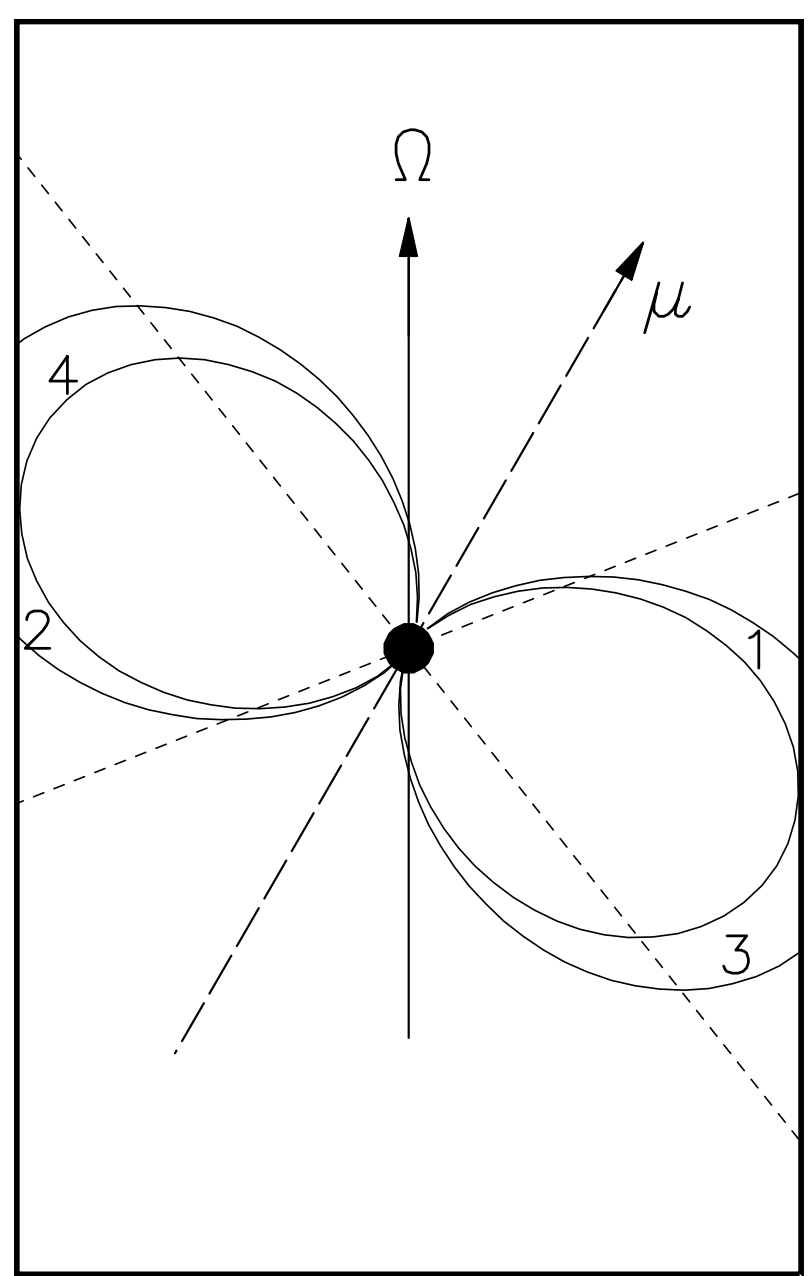

FIG. 1.-Schematic illustration of the outer gaps of a pulsar. The outer gap extends from the null charge surfaces (dashed lines) to the light cylinder. The value $r_{\text {in }}$ is the distance from the star to the intersection between the null charge surfaces of gap 1(2) and the first open field lines.

meaningful phase-resolved study of their emissions (Fierro 1995; Thompson et al. 1996; Fierro et al 1998). For the three brightest, high-energy $\gamma$-ray pulsars (Crab, Vela, and Geminga), Fierro et al. (1998) introduced a "hardness ratio," ratio $\equiv F(>300 \mathrm{MeV}) / F(100-300 \mathrm{MeV})$, to characterize the evolution of the spectral index as a function of pulsar phase, where $F(>300 \mathrm{MeV})$ and $F(100-300 \mathrm{MeV})$ are the maximum likelihood fluxes measured above 300 $\mathrm{MeV}$ and between 100 and $300 \mathrm{MeV}$, respectively. Their analysis indicated that (1) two peaks are observed and they are not symmetric; (2) the phase-resolved spectra are different at various phase intervals; (3) the hardest emission from $\mathrm{Crab}$ and Vela pulsars results from the bridge region between the two $\gamma$-ray peaks, while the hardest emission from the Geminga pulsar comes from the second $\gamma$-ray peak; and (4) the phase-resolved spectra of the pulsed components of all three $\gamma$-ray pulsars have approximately the same energy turnover in each component, although there is a large variation in the spectral index over the phase interval. These observed facts provide strong constraints on theoretical models for $\gamma$-ray pulsars. The comparison of the CHR model with EGRET observations indicates that some of the morphological features of emission expected in the CHR model appear to be inconsistent with the EGRET observations. For example, Crab and Geminga obser- vations indicate that (1) those pulsars have peaks which are not strongly cusped and have both strong bridge emission and significant off-pulse emission and (2) the two pulses are of roughly similar strength. Chiang \& Romani $(1992,1994)$ reconsidered and modified a CHR-type model for a threedimensional magnetosphere. They assumed that outer gaps exist along all field lines on the boundary of the closed region instead of just on the bundle of field lines lying close to the plane defined by the rotation and magnetic dipole axes. In a static magnetic field approximation, they showed that assuming a single pole gave only outgoing "curvature radiation" particle flow which is the same on each field line through the null surface produced a broad, irregularly shaped emission beam which is particularly intense near its edge. Then two $\gamma$-ray peaks (subpulses) will be observed when the line of sight from the Earth crosses these enhanced regions of the $\gamma$-ray beam, while the inner region of the beam provides a significant amount of emission between the peaks. A wide range of peak phase separations can be accommodated with a proper choice of the observer colatitude. Further, Romani \& Yadigaroglu (1995, hereafter RY) developed a model for the beaming of high-energy $\gamma$-ray emission from a single outer gap of a rotating inclined dipole. They assumed that almost no emission is beamed inward along the field lines and the field lines are those which would exist if there were no charged particles flow in the magnetosphere. Although no detailed spectra or luminosities have been calculated for this model, their studies help in modeling the phase emission features. Zhang \& Cheng (1997) proposed a self-consistent model for $\gamma$-ray pulsar emission. In their model, a criterion for the existence of an outer gap is given in terms of the fractional size of the outer gap, $f=5.5 P^{26 / 21} B_{12}^{-4 / 7}$, where $P$ is the period in seconds and $B_{12}$ is the magnetic field in units of $10^{12} \mathrm{G}$. If $f>1$, a self-sustained outer gap accelerator does not exist.

In this paper, we reconsider the three-dimensional magnetosphere, following the important groundbreaking work of Romani and coworkers. But, instead of assuming a single outer gap with only outgoing current and no restriction on azimuthal directions, we use various physics processes (especially pair production which depends sensitively on the local electric field and the local magnetic field's radius of curvature, surface field structure, reflection of $e^{ \pm}$pairs because of mirroring and resonant X-ray electron scattering) to determine the three-dimensional geometry of the outer gap. In our model, two outer gaps and both outgoing and incoming currents are in principle allowed, but it turns out that outgoing currents dominate the emitted radiation intensities. (A single outer gap geometry may be generally more appropriate for a strongly offset dipole moment where, although the incoming current is strong, the related incoming radiation is restricted to a relatively small region of the outer magnetosphere.) In $\S 2$ we calculate both static and rotating three-dimensional structures of the magnetosphere. In $\S 3$ we study pair production conditions and determine the three-dimensional structure of an outer gap. In $\S 4$ we examine various morphologies for $\gamma$-ray emission with calculated emission patterns and pulse profiles. The phase-resolved spectra for the Crab pulsar are calculated in $\S 5$. A final discussion is given in $\S 6$.

\section{THE GEOMETRY OF THE MAGNETOSPHERE}

In a static dipolar magnetic field approximation, basic features of the pulsar magnetosphere can be understood. 


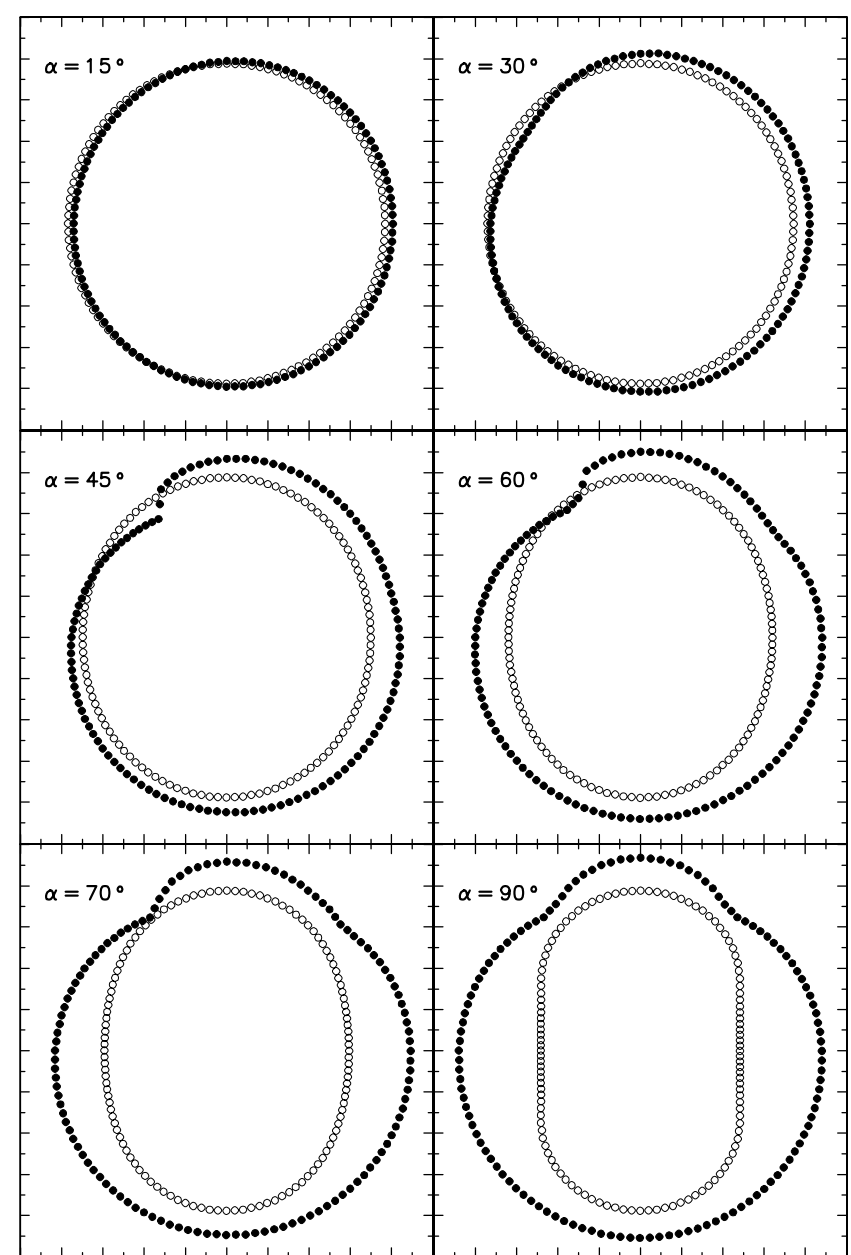

FIG. 2.-Polar cap shapes for various inclination angles in both static and rotating magnetosphere approximations. The minor axis of the static dipole case is in the $(\Omega, \mu)$-plane.

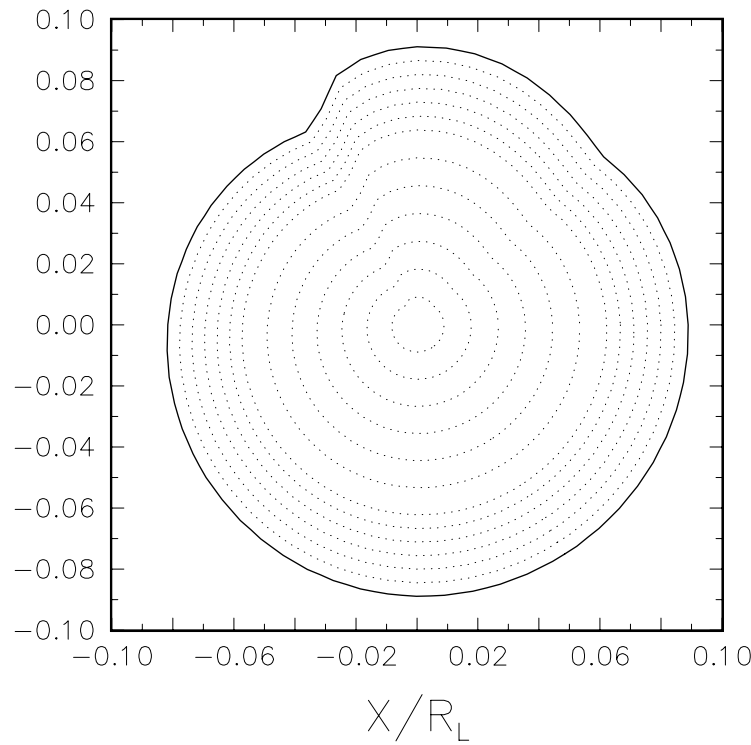

FIG. 3.-Example of a coordinate grid for the open volume. We define that $a_{1}=1.0$ for the last closed line; $a_{1}$ changes by 0.05 from 1.0 to 0.7 and 0.1 from 0.7 to 0.1 .
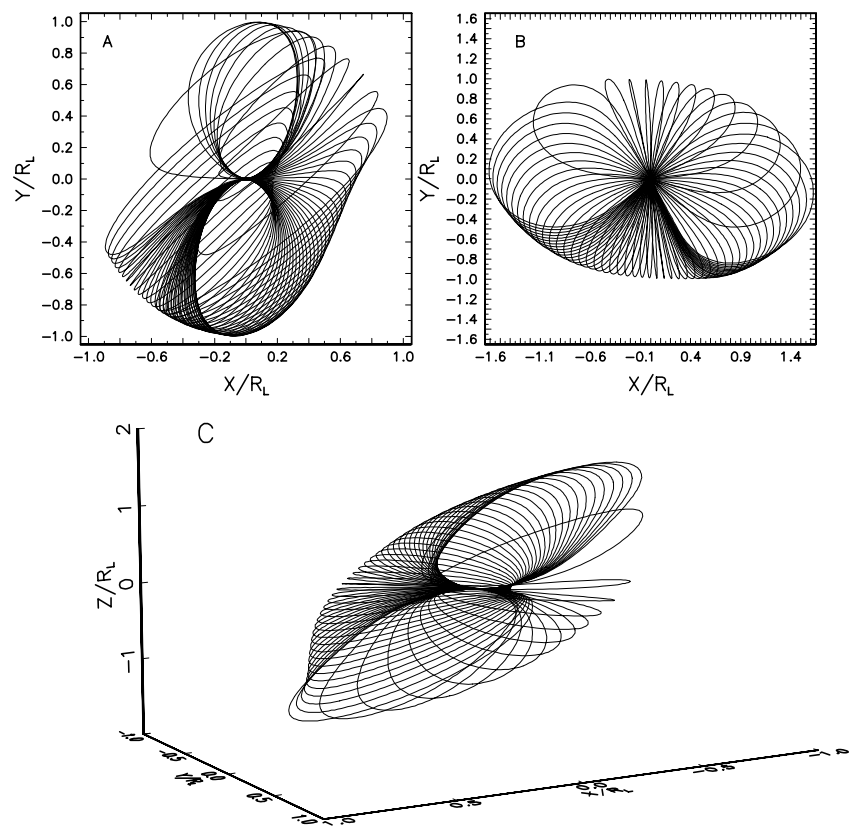

FIG. 4.- Last closed field lines for a dipole inclined to the rotation axis with angle $\alpha=65^{\circ}$. (a) A projection looking down the rotation axis. (b) A projection looking down the magnetic axis. (c) A three-dimensional view.

However, it is of course more realistic that the magnetosphere be approximated as a rotating inclined magnetic dipole. The magnetic field lines of a rotating inclined dipole are especially different from those of a static dipole for large inclination angles. In this section, we describe both static and rotating oblique dipole fields.

\subsection{Dipole Field}

For a static oblique dipole, the magnetic field

$$
\boldsymbol{B}=\frac{1}{r^{3}}[3(\mu \cdot \hat{\boldsymbol{r}}) \hat{\boldsymbol{r}}-\mu],
$$

where $\mu$ is the magnetic moment vector and $\hat{\boldsymbol{r}}$ is the radial unit vector. The needed static dipole field of a pulsar magnetosphere in Cartesian coordinates is given in Appendix A.

For an inclined dipole of constant magnitude, rotating with angular frequency $\Omega$ about the $\hat{z}$-axis, the magnetic moment is

$$
\mu(t)=\mu(\sin \alpha \cos \Omega t \hat{x}+\sin \alpha \sin \Omega t \hat{y}+\cos \alpha \hat{z}),
$$

where $\alpha$ is the inclination angle of $\mu$ to $\hat{z}$. The vacuum magnetic field is (e.g., Jackson 1975; Yadigaroglu 1997)

$$
\begin{aligned}
\boldsymbol{B}= & -\left[\frac{\mu(t)}{r^{3}}+\frac{\dot{\mu}(t)}{c r^{2}}+\frac{\ddot{\mu}(t)}{c^{2} r}\right] \\
& +\hat{r} \hat{r} \cdot\left[3 \frac{\mu(t)}{r^{3}}+3 \frac{\dot{\mu}(t)}{c r^{2}}+\frac{\ddot{\mu}(t)}{c^{2} r}\right],
\end{aligned}
$$

where $\hat{r}=r / r$. The needed three components of the rotating dipole field in Cartesian coordinates are given in Appendix B (eqs. [B2]-[B4]) for an empty magnetosphere. Unfortunately, the needed ones for a realistically "loaded" one are still unavailable. The needed part of the structure of the magnetosphere can still only be described by numerical integrals. Here we are interested in specific features such as the last closed field lines. Following Arendt \& Eilek (1998), 
we searched for the solutions by using Runge-Kutta integration. The last closed field lines are found as follows: (1) give a set of initial values at the stellar surface, (2) use the Runge-Kutta integration to follow a particular field line in space and determine whether this line closes inside the light cylinder or crosses it, and (3) iterate on the initial value until the field line is just tangential to the light cylinder.

\subsection{Some Numerical Results}

In this section, we give some calculated results for static and rotating magnetospheres. The pulsar magnetosphere can be divided into two zones: one is "closed" with plasma corotating with the star and the other is the "open " zone in which plasma flows along the open field lines and escapes through the light cylinder. The last closed field lines are the borders of the open magnetic field line bundle. The accelerators (both polar gap and outer gap) are within this open zone. The footprints of the last closed field lines on the stellar surface define the polar cap shape. In the static approximation, the polar cap shape is a circle with radius of $R_{\mathrm{pc}}=R\left(R / R_{L}\right)^{1 / 2}$ for an aligned dipole (e.g., Sturrock 1971), where $R_{L} \equiv c \Omega^{-1}$; as the inclination angle $\alpha$ increases, the shape becomes more elliptical, especially compressed in the latitude direction by an amount up to $\sim 60 \%$ for an orthogonal dipole (Roberts \& Sturrock 1972; Biggs 1990). However, for the rotating dipole the polar cap shape is different from that for a static dipole, especially at large inclination angles. Because the polar cap shape is sensitive to the shape of the magnetic field lines close to the light cylinder and the field lines for a nonaligned rotating dipole are strongly distorted close to the light cylinder, these highlatitude distortions give a polar cap shape that is neither circular nor simply elongated (RY; Arendt \& Eilek 1998). We use Crab pulsar parameters to show the polar cap shapes for different magnetic inclination angles in Figure 2. This polar cap shape defines the boundary of the open volume at the stellar surface. Because the outer gaps are within the open volume, we divide the open volume into many parts, in which the shape of each part at the stellar surface is the same as the polar cap shape but the size is smaller. First, we determine the coordinate values $\left(x_{0}, y_{0}, z_{0}\right)$ of the last closed field lines at the stellar surface. Then the coordinate values $\left(x_{0}^{\prime}, y_{0}^{\prime}, z_{0}^{\prime}\right)$ for different parts can be determined by using $x_{0}^{\prime}=a_{1} x_{0}, y_{0}^{\prime}=a_{1} y_{0}$, and $z_{0}^{\prime}=\left[1-\left(x_{0}^{\prime 2}+y_{0}^{\prime 2}\right)\right]^{1 / 2}$ and changing $a_{1}$. In Figure 3 , the open volume divided into these many parts is shown.

In Figure 4, the three-dimensional structure of the last closed field lines for the rotating dipole with $\alpha=65^{\circ}$ is shown. In our calculations, but not those of Arendt \& Eilek (1998), Crab pulsar parameters are used and the azimuth around the magnetic axis is uniformly divided into 60 segments.

\section{PAIR PRODUCTION AND THE STRUCTURE OF OUTER GAPS}

In CHR I, four outer gaps exist in the open zone in the plane of $(\boldsymbol{\Omega}, \mu)$ (two of them are topologically connected in three-dimensional space), but only the two longer outer gaps give observed fan beams. They argued that these two longer outer gaps may create enough $\gamma$-ray and $e^{ \pm}$pairs to quench the two shorter, less powerful ones. In the CHR model, the $\gamma$-ray emission is approximated to occur only along the last closed field line in the plane of the dipole and rotation axes. Because both positive and negative charged particles are accelerated in the gap, the emission should beam both toward and away from the pulsar. Therefore, the observed fan beams consist of those coming from different gaps. In the single outer gap model (Chiang \& Romani 1992, 1994; RY), however, the emission is assumed to occur along all field lines on the boundary of the closed region, and the observed beams are assumed to be produced from various parts of a single outer gap. In order to avoid the appearance of several peaks of emission in their model, it was assumed that the emission inward in the gaps is negligible. Important questions are (1) how many outer gaps exist in the open zone and (2) why the emission inward can/ cannot be ignored. In subsequent sections, we show that (1) pair creation inside the outer gap is restricted in both the longitudinal direction (along the magnetic field lines) and the transverse direction (in the $\phi$-direction) because of local photon-photon pair creation criteria; (2) most curvature photons are converted into secondary $e^{ \pm}$pairs above the outer gap, including those regions with only outgoing current; (3) the inactive parts of the outer gap will be filled with secondary $e^{ \pm}$pairs resulting from effects of X-ray resonant scattering; and (4) a single outer gap in the magnetosphere is a viable possibility.

\subsection{Pair Production inside the Outer Gap}

For a neutron star with a strong magnetic field $\boldsymbol{B}$ and angular velocity $\Omega$, the charge density of the magnetosphere in the corotating frame of the neutron star is (Goldreich \& Julian 1969)

$$
\rho_{0}=-\frac{\boldsymbol{\Omega} \cdot \boldsymbol{B}}{2 \pi c}[1+O(|\boldsymbol{\Omega} \times \mathbf{r}| / c)] .
$$

From equation (4), the charge density changes sign when a global current flows through the null surface $(\boldsymbol{\Omega} \cdot \boldsymbol{B}=0)$. As a result, a strongly charge-deficient region $(\rho \approx 0)$ in the outer magnetosphere around the null surface will form if there is charge-separated flow. A deviation of the charge density from $\rho_{0}$ results in an electric field along $\boldsymbol{B}$. If this electric field becomes strong enough to accelerate $e^{ \pm}$pairs to ultrarelativistic energies, $e^{ \pm}$pairs could radiate $\gamma$-ray tangential to the curved $\boldsymbol{B}$ field lines there. These "curvature $\gamma$-rays" may be converted into $e^{ \pm}$pairs via $\gamma+\gamma \rightarrow e^{+}+e^{-}$or $\gamma+\mathrm{X} \rightarrow e^{+}+e^{-}$. In order to keep a steady current flow and the charge density $\rho_{0}$ in the regions outside the gap, the gap will grow until it is large enough and the electric field is strong enough to maintain a sufficiently copious supply of charges to the rest of the open field line region. If the gap ends in a region where $\rho_{0} \neq 0$ and $\boldsymbol{E} \cdot \hat{\boldsymbol{B}} \neq 0$, charges from the surrounding region may flow in through the end. If both ends are located on the null surface, $e^{ \pm}$produced in the gap will replace the charge deficiency inside the gap, and finally the gap will fill up. However, if a vacuum gap extends to the light cylinder, charged particles created in the gap should escape from the magnetosphere, so the gap would not be quenched. Hence, stable outer gaps (if they exist) are those from the null surface to the light cylinder along the last closed field lines. In each outer gap, the inner boundary of a thin outer gap lies near the intersection of the null surface where $\rho_{0}=0$ and the boundary of the closed field lines of the star on which the magnetosphere current does not flow. The thickness of the outer gap is bounded from above by a layer of electric current which contributes a surface charge density (CHR I). 
In order to estimate the three-dimensional structure of an outer gap, we follow the self-consistent pair production scheme proposed by Zhang \& Cheng (1997, hereafter ZC) instead of that proposed in the CHR model, which is basically a two-dimensional model, and the crossed-beam assumption may not be valid everywhere along the outer gap. In the ZC model, the characteristic photon energy is determined by the size of the outer gap $\left(f_{0}\right)$ and is given by $E_{\gamma}^{\text {sc }}\left(f_{0}\right) \approx 2 \times 10^{8} f_{0}^{3 / 2} B_{12}^{3 / 4} P^{-7 / 4} \mathrm{eV}$, where the radius of the neutron star is assumed to be $10^{6} \mathrm{~cm}$. Half of the primary $e^{ \pm}$pairs in the outer gap will move toward the star and lose their energy via the curvature radiation. The return particle flux is approximately $\dot{N}_{e^{ \pm}} \approx \frac{1}{2} f_{0} \dot{N}_{\mathrm{GJ}}$, where $\dot{N}_{\mathrm{GJ}}$ is the Goldreich-Julian particle flux (Goldreich \& Julian 1969). Although most of the energy of the primary particles is lost on the way to the star via curvature radiation, about $10.6 P^{1 / 3}$ ergs per particle will still remain to be finally deposited on the stellar surface. That energy will be emitted as X-rays from the stellar surface (Halpern \& Ruderman 1993). The characteristic energy of these $X$-rays is $E_{\mathrm{X}}^{h} \approx$ $3 k T \approx 1.2 \times 10^{3} P^{-1 / 6} B_{12}^{1 / 4} \mathrm{eV}$. The $\mathrm{KeV} \mathrm{X}$-rays from a hot polar cap may be reflected back to the stellar surface by cyclotron resonance scattering if there is a large density of $e^{ \pm}$pairs near the neutron star surface (Halpern \& Ruderman 1993; Zhu \& Ruderman 1997) and eventually reemitted as soft $\mathrm{X}$-rays with characteristic temperature

$$
T_{s} \approx 3.8 \times 10^{5} f_{0}^{1 / 4} P^{-5 / 12} B_{12}^{1 / 4} \xi^{1 / 4} .
$$

Every pair produced by X-ray and curvature photon collisions in the outer gap can emit $10^{5}$ photons in it. Such huge multiplicity can produce a sufficient number of $e^{ \pm}$ pairs to sustain the gap as long as the center of mass energy of colliding X-ray and curvature photons is higher than the threshold energy for electron/positron pair production, i.e., $E_{\mathrm{X}} E_{\gamma}^{\mathrm{sc}}\left(f_{0}\right) \geq\left(m_{e} c^{2}\right)^{2}$. Because of this photon-photon pair production, the size of the outer gap limited by the soft thermal X-rays from the neutron star surface is

$$
f_{0} \approx 5.5 P^{26 / 21} B_{12}^{-4 / 7} \xi^{-1 / 7},
$$

where $\xi=\Delta \Phi / 2 \pi$. Here $\Delta \Phi$ is the transverse extension of the outer gap and $f_{0}=h_{0} / R_{L}$ with $h_{0}$ the average vertical extension (perpendicular to the magnetic field) of the outer gap. In the three-dimensional case, $h_{0}$ is the average separation between two equipotential surfaces which define the boundary of the outer gap. In the ZC model, $\xi$ was chosen to be unity. Most of the soft photons in the Crab pulsar are emitted by the secondary pairs produced by the collision between curvature photons from the outer gap and synchrotron X-rays (CHR II). In other words, the Crab pulsar is self-controlled by its own secondary synchrotron photons. In CHR II, they have taken into account the effect of soft photons emitted by secondary pairs and showed that the fractional size is given by $33.2 B_{12}^{-13 / 20} P^{33 / 20}$. Using the parameters of the Crab pulsar, CHR estimated that the fractional size of the gap is 0.05 . On the other hand, equation (6) gives 0.04 . However, the estimate of the fractional size of the outer gap in the three-dimensional case in terms of these secondary synchrotron photons is very difficult (Romani 1996) since the estimate from the two-dimensional model with the effect of secondary synchrotron photons is very close to that of equation (6). So here we consider equation (6) as an estimate of the structure of an outer gap. The dependence on the unknown $\xi$ is weak. On the other hand, if the inclination angle is very large, the inner boundaries of outer gaps 1 and 2 will move close to the neutron star surface. In this case, magnetic pair production may be important. Using the criterion for magnetic pair production (e.g., Ruderman \& Sutherland 1975), $\left(E_{\gamma} / 2 m c^{2}\right)\left(B \theta / B_{q}\right) \geq$ $1 / 15$ with $\theta \sim l / s \sim\left(2 f_{B}\right)^{1 / 2}\left(r_{\text {in }} / R_{L}\right)^{3 / 2}$ and $B_{q}=4.4 \times 10^{13}$ $\mathrm{G}$, we have

$$
f_{B}\left(R_{L} / 2\right) \approx 30.8 B_{12}^{-7 / 8} \Omega_{2}^{-19 / 8}(\tan \alpha)^{-21 / 8},
$$

where $\Omega_{2}=\Omega / 10^{2} \mathrm{rad} \mathrm{s}^{-1}$. For the Crab parameters, $f_{B}\left(R_{L} / 2\right) \sim 1.82(\tan \alpha)^{-21 / 8}$. Therefore, we suggest that gap geometry will not be changed unless the inclination angle is greater than $\sim 80^{\circ}$.

There are two methods to estimate $\xi$. It has been shown (Halpern \& Ruderman 1993; ZC; Cheng, Gil, \& Zhang 1998; Wang et al. 1998) that half of the charged particles produced in gap 1 and gap 2 will move toward the star and lose their energy via curvature radiation. Part of the highenergy curvature photons will be converted into secondary $e^{ \pm}$pairs in the field lines which connect to gaps 3 and 4 via either photon-photon pair creation or magnetic pair production. Part of the incoming secondary pairs created in low magnetic field regions may be reflected back and enter gaps 3 and 4 (Ho 1986). Those secondary pairs created in strong magnetic field regions will lose their energies via synchrotron radiation, but some of these lower energy secondary pairs will be reflected back by the resonant X-ray scattering near the stellar surface (Wang et al. 1998) and also enter gap 3 and gap 4 . Therefore, the reflected outgoing $e^{ \pm}$pairs can enter gaps 3 and 4 to quench them. In this case, the extension along the azimuthal direction is about $180^{\circ}$, corresponding to a maximum value of the inner boundary of the outer gap of $r_{\text {in }}^{\prime}$ where the null charge surface intercepts the light cylinder.

Another method of estimating $\xi$ is to consider the local pair production condition because the energy of a curvature photon $\left(E_{\gamma}\right)$ depends on the local electric field which is a function of position. In the $(\Omega, \mu)$-plane along the last closed field lines, for example, $E_{\gamma} \propto x^{-13 / 4}$ in a thick outer gap (ZC) and $E_{\gamma} \propto x^{-1 / 4}$ in a thin outer gap (CHR I), where $x=s / R_{L}$ with $s$ the radius of curvature and $R_{L}$ the radius of the light cylinder. Generally, we have

$$
E_{\gamma} \propto x^{-\alpha_{E}}
$$

with $\alpha_{E}>0$. Because $E_{\gamma}$ decreases with increasing $x$, the pair production region will be limited to a small part of the entire outer gap region. The cross section for photonphoton pair production, assuming a head-on collision, is

$$
\sigma_{\gamma \gamma}=\frac{3 \sigma_{T}}{16}\left(1-v^{2}\right)\left[\left(3-v^{4}\right) \ln \left(\frac{1+v}{1-v}\right)-2 v\left(2-v^{2}\right)\right],
$$

where $1-v^{2}=\left(m_{e} c^{2}\right)^{2} / \epsilon E_{\gamma}$ and $\epsilon$ is the energy of the soft photon. For a given $\epsilon$ (in the ZC model, $\epsilon \sim k T_{s}$ ), the dependence of $E_{\gamma}$ on $\sigma_{\gamma \gamma}$ is known. In order to determine the pair production both along the magnetic field line direction and over the $\phi$-direction, we need to consider the local pair creation conditions

$$
\begin{aligned}
N_{e^{ \pm}}(r) & =\left(1-e^{-\tau_{\gamma \gamma}(r)}\right) N_{\gamma}(r) \approx \tau_{\gamma \gamma} N_{\gamma}(r), \\
N_{\gamma}(r) & =\frac{e E_{||}(r)}{E_{\gamma}(r)},
\end{aligned}
$$

where $N_{e^{ \pm}}$is the number of $e^{ \pm}$pairs produced at radius $r$ per electron/positron per unit length and $N_{\gamma}$ is the number of curvature photons emitted at $r$ per unit length. The value 
$E_{||}(r)$ is the local electric field along the magnetic field, $E_{\gamma}(r)$ is the characteristic energy of the local curvature photons, $\tau_{\gamma \gamma}=n_{x}(r) \sigma_{\gamma \gamma}\left(T_{s}, E_{\gamma}\right) l(r)$ is the optical depth of the photonphoton pair creation, $n_{x}=4 \pi R^{2} \sigma T_{s}^{4} / 4 \pi r^{2} k T_{s} c, l(r) \approx$ $\left[2 s(r) f(r) R_{L}\right]^{1 / 2}$ is the optical path of the curvature photons within the outer gap, $f(r)=h(r) / R_{L}$, and $h(r)$ is the local vertical extension of the outer gap [since $h^{2}(r) B(r)=$ constant, $f(r) \propto r^{3 / 2}$, and $\left.f_{0} \sim f\left(r=R_{L} / 2\right)\right]$. For a thin outer gap (e.g., the Crab pulsar), $E_{||} \propto r^{-1 / 2}$ (CHR I) and $E_{\gamma} \propto r^{-1 / 8}$. With such weak dependence of $E_{\gamma}(r)$ on $r$, we assume that $\sigma_{\gamma \gamma} \approx$ constant over the pair production region. Therefore, $\tau_{\gamma \gamma} \propto r^{-1}, N_{\gamma}(r) \propto r^{-3 / 8}$, and the total number of $e^{ \pm}$pairs produced at $r$ per electron/positron $r N_{e^{ \pm}} \propto r^{-3 / 8}$. We see that most pairs will be produced near the null surface. To estimate the radial extension within which pair production takes place, we require

$$
\frac{r_{\mathrm{lim}}^{\prime} N_{e^{ \pm}}\left(r_{\mathrm{lim}}^{\prime}\right)}{r_{\mathrm{in}} N_{e^{ \pm}}\left(r_{\mathrm{in}}\right)} \sim\left(\frac{r_{\mathrm{lim}}^{\prime}}{r_{\mathrm{in}}}\right)^{-3 / 8} \sim \frac{1}{2} .
$$

Therefore, pair production processes mainly take place between $r_{\text {in }}$ and $r_{\text {lim }}^{\prime} \sim 6 r_{\text {in }}$ and $\Delta \Phi\left(r_{\text {lim }}\right) \sim 160^{\circ}$ (cf. Fig. $5 b$ ). For a thick outer gap, $E_{\gamma} \propto r^{-13 / 8}$. Such strong dependence of $E_{\gamma}$ on $r$ will make the cross section for pair production decrease rapidly. Since pair production must take place in the beginning of the outer gap (otherwise there is no current passing through the outer gap), we argue that the radial extension of the outer gap must be

$$
r_{\text {lim }}^{\prime} \approx f_{0} R_{L}
$$

and $\Delta \Phi \sim \pi$ for Geminga parameters.

Therefore, in either a thin or a thick outer gap the pair production region inside the outer gap is in the region from $r_{\text {in }}$ to $r_{\text {lim }}$, where

$$
r_{\text {lim }}=\min \left(r_{\mathrm{in}}^{\prime}, r_{\text {lim }}^{\prime}\right) \text {. }
$$

The extension along the azimuthal direction is

$$
\Delta \Phi=\min \left[180^{\circ}, \Delta \Phi\left(r_{\text {lim }}^{\prime}\right)\right] .
$$

In this model, pair production takes place near the null surface, especially for a large magnetic inclination angle. This implies that (1) only outgoing particle flow exists in front of the outer gap region (in agreement with the assumption of RY) and (2) the azimuthal extension of the outer gap is less than $\pi$. In calculating the emission morphologies $(\S 4)$, light curves with double-peak structures are the most common even though two outer gaps exist.

Although we have argued that the pair production processes inside the outer gap only exist in a region close to the null surface, the pair production region outside the outer gap can extend all the way to the light cylinder, at least, for the Crab pulsar. Those pairs created outside the outer gap will lose their energies via $\mathrm{MeV}$ to optical synchrotron radiation. In the CHR model, these lower energy photons do not pass through the outer gap, but they can collide with the high-energy curvature photons from it and produce $e^{ \pm}$pairs. In our model, the synchrotron X-rays from secondary pairs and the curvature photons will collide at an angle $\sim 2 f_{0} \sim 0.1$ for the Crab pulsar; the mean free path is $\lambda_{\mathrm{X} \gamma} \sim\left[L_{\mathrm{X}}\left(2 f_{0}\right) E_{\gamma} \sigma_{p} / l^{2} c(\mathrm{MeV})^{2}\right]^{-1} \sim$ $10^{7}\left(f_{0} / 0.1\right)^{-1} L_{36}^{-1} l_{8}^{2}\left(E_{\gamma} / 10 \mathrm{GeV}\right)^{-1} \mathrm{~cm}$, where $L_{36}$ is the $\mathrm{X}$-ray luminosity in units of $10^{36} \mathrm{ergs} \mathrm{s}^{-1}, l_{8}$ is the length of the outer gap in units of $10^{8} \mathrm{~cm}, E_{\gamma}$ is the typical energy of curvature radiation photons, and $\sigma_{p}$ is the pair production
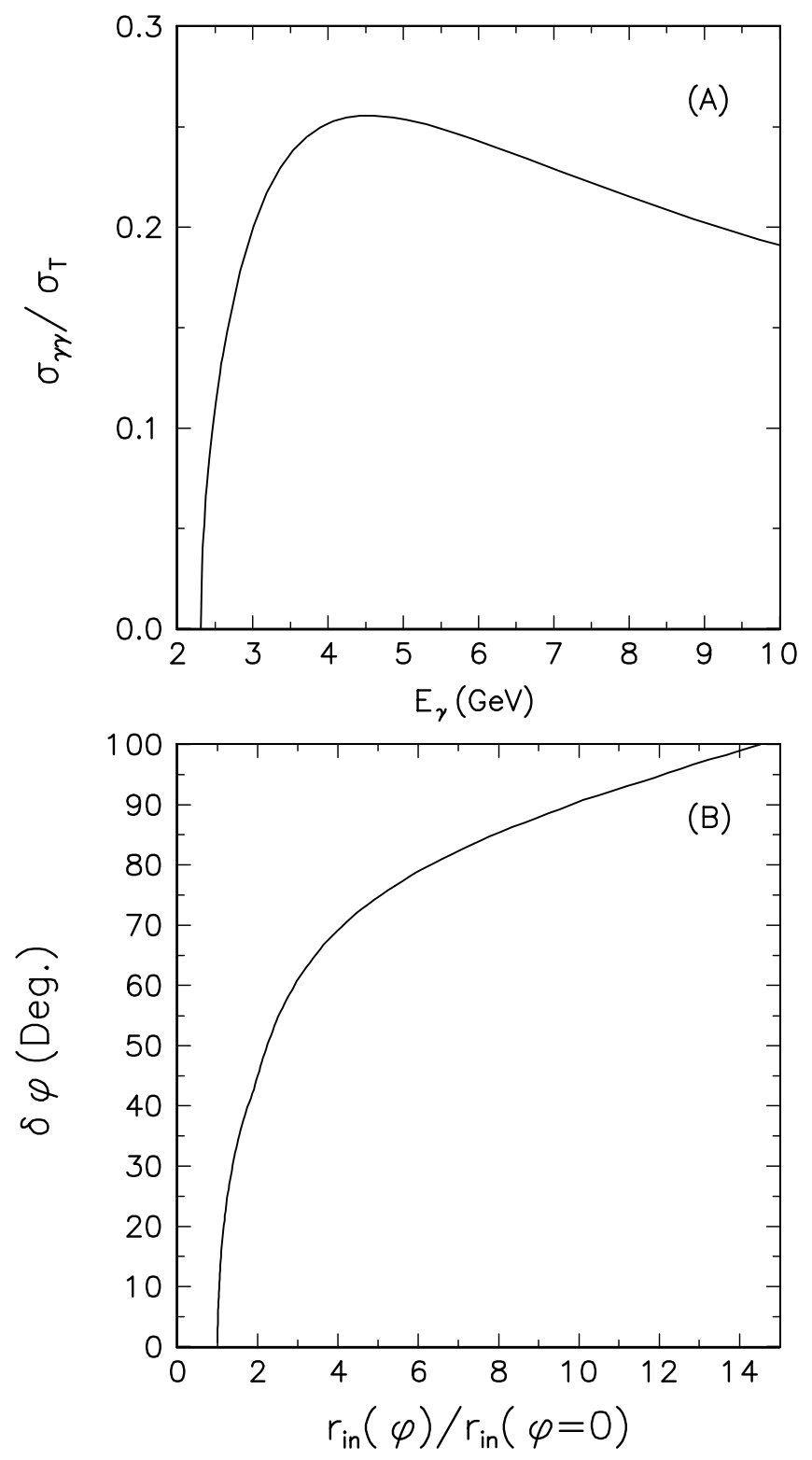

FIG. 5.-(a) $E_{\gamma}$ vs. the azimuth $\phi$ [perpendicular to the $(\Omega, \mu)$-plane] for $\alpha=65^{\circ}$. The typical energy of soft photons is assumed to be $100 \mathrm{eV}$. (b) Azimuthal $\phi$ vs. inner boundary radius $r_{\text {in }}$ of the outer gap for $\alpha=65^{\circ}$.

cross section. So, most curvature photons emitted from the accelerator will become secondary pairs outside the gap for the Crab pulsar. However, this certainly should not be the case for $\gamma$-ray pulsars such as Geminga because there are not enough $\mathrm{X}$-rays around the outer gap.

\subsection{Structure of the Outer Gap}

As pointed out above, two outer gaps can exist in the open field line volume of the pulsar magnetosphere in this model. For each outer gap, the region of the outer gap along the radial direction is from the inner boundary $r_{\text {in }}$ to the radius of the light cylinder $\left(R_{L}\right)$, and the extension of the outer gap along the azimuthal direction $(\Delta \phi)$ is limited by the photon-photon production. The gap thickness can be described by the fractional size $f$ of the outer gap (CHR I; CHR II; Ho 1989; ZC). Moreover, the photon-photon pair production inside the outer gap can occur only in the region $\left(r_{\text {lim }}-r_{\text {in }}\right)$ along the radial direction. Therefore, outward 
emission can exist in the whole outer gap, but the inward emission is only from the region $\left(r_{\text {lim }}-r_{\text {in }}\right)$. The inner boundary $r_{\text {in }}$ of the outer gap is the intersection of the null charge zone with the last closed field line determined by $\boldsymbol{\Omega} \cdot \boldsymbol{B}=0$, which will vary with azimuth. In the $(\Omega, \mu)$-plane, $r_{\text {in }} / R_{L} \sim$ $4 / 9 \tan ^{2} \alpha$ for $\alpha \geq 45^{\circ}$ (see, for example, Halpern \& Ruderman 1993) or $r_{\text {in }} / R_{L} \sim 4(\pi / 2-\alpha)^{2} / 9$ (Romani 1996).

\subsection{Possibility of a Single-Pole Outer Gap}

In several models (e.g., Ruderman 1991a, 1991b, 1991c), the surface magnetic field of some pulsars may have a sunspot-like geometry. Such an off-center dipole implies that one of the outer gaps should be deactivated by polar gap activity because some of the high-energy photons emitted by many polar gap primary charged particles will be deflected by the gravitational field of the star and be converted to pairs on the magnetic field lines which are connected with one of the two outer gaps (cf. Ruderman 2000). The maximum angle of the deflection is

$$
\delta \Phi \sim \frac{l}{R} \frac{G M}{R c^{2}},
$$

where $l$ is now the thickness of the crust, $M$ the mass of the star, and $R$ its radius. This effect is not limited to a sunspotlike dipolar field geometry and/or light deflection by gravity. For example, strong multipole fields can give surface magnetic field lines bending such that some photons emitted tangentially to field lines in the polar cap accelerator will make $e^{ \pm}$pairs flowing away on field lines leading to the outer gap accelerator and quench it. Therefore, in general a single outer gap accelerator (or even none) could be a common structure in the outer magnetosphere of pulsars whose $\boldsymbol{\Omega}$ and $\mu$ are otherwise sufficient to sustain outer gap accelerators.

\section{PHOTON EMISSION MORPHOLOGIES}

We now consider the morphological features of emission from outer gaps. Chiang \& Romani (1994) and RY assumed the entire locus of points on the last closed surface bounded by the null surface and the light cylinder as giving emission in an outer gap. In our model, the emission-producing outer gaps are limited along both the radial and the $\phi$-directions. For example, the extension of the outer gap on the $\phi$ direction is about $160^{\circ}$ for Crab parameters with $\alpha=65^{\circ}$. We describe the new photon emission morphologies below.

\subsection{The Projection of Photon Emissions in the $(\zeta, \Phi)$-Plane}

High-energy photons will be emitted nearly tangent to the magnetic field lines in the corotating frame because of the relativistic $1 / \gamma$ beaming inherent in high-energy processes unless $|\boldsymbol{E} \times \boldsymbol{B}| \sim B^{2}$. Then following RY, we assume relativistic charged particles in the open zone radiate in their direction of propagation, i.e., along the magnetic field lines in the corotating frame. For each location within the open zone, the direction of emission expressed as $(\zeta, \Phi)$ is calculated, where $\zeta$ is the polar angle from the rotation axis and $\Phi$ is the phase of rotation of the star. Effects of travel time and aberration are taken into account. A photon with velocity $\boldsymbol{u}=\left(u_{x}, u_{y}, u_{z}\right)$ along a magnetic field line with a relativistic addition of velocity along the azimuthal angle gives an aberrated emission direction $\boldsymbol{u}^{\prime}=\left(u_{x}^{\prime}, u_{y}^{\prime}, u_{z}^{\prime}\right)$. Travel time gives a change of the phase of the rotation of the star. Combining these two effects, and choosing $\Phi=0$ for radiation in the $(x, z)$-plane from the center of the star, $\zeta$ and $\Phi$ are given by (Yadigaroglu 1997)

$$
\begin{aligned}
\cos \zeta & =u_{z}^{\prime}, \\
\Phi & =-\phi_{u^{\prime}}-\overrightarrow{\boldsymbol{r}} \cdot \hat{u}^{\prime},
\end{aligned}
$$

where $\phi_{u^{\prime}}$ is the azimuthal angle of $\hat{u}^{\prime}$ and $\overrightarrow{\boldsymbol{r}}$ is the emitting location in units of $R_{L}$. We project photon emissions on the $(\zeta, \Phi)$-plane and observe the emission patterns on the sky. In the $(\zeta, \Phi)$-plane, the null surface can be determined easily because it consists of the points at which magnetic field lines are perpendicular to the rotation axis. For a field line, the null charge crossing is where the projected line crosses the equatorial line $\left(\zeta=90^{\circ}\right)$. As examples, we show the projections of photon emission both outward and inward from two outer gaps in the $(\zeta, \Phi)$-plane for different magnetic inclination angles $\left(\alpha=30^{\circ}, 45^{\circ}, 65^{\circ}\right.$, and $\left.80^{\circ}\right)$ in Figure 6, where the extension of the outer gaps on the $\phi$-direction is assumed to be $180^{\circ}$ and $\left(r_{\text {lim }}-r_{\text {in }}\right) \sim 0.55 R_{L}$.

\subsection{Emission outward from the Outer Gap}

As mentioned above, the pulse profile depends on emission location and viewing angle. Here we use Crab param-

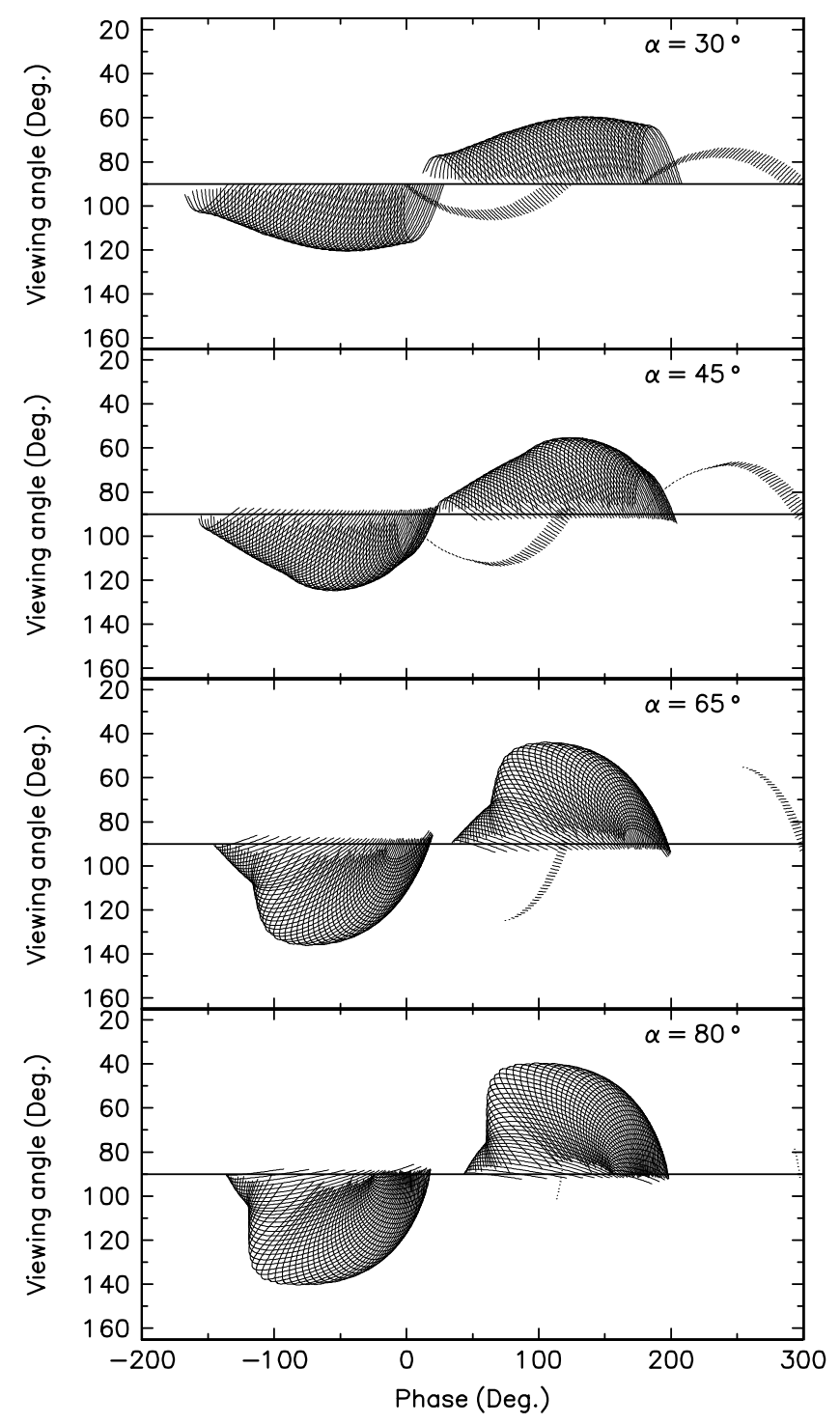

FIG. 6.-Emission projections onto the $(\zeta, \Phi)$-plane for various magnetic inclination angles. The photons emitted both outward and inward from two outer gaps are shown. Crab pulsar parameters are used. 
eters and $\alpha=65^{\circ}$ to describe the outward emission from an outer gap. For simplicity, we assume that the photon emissivity is uniform. For comparison, we at first consider the model proposed by Chiang \& Romani (1994) and RY, i.e., a single-pole outer gap. In their model, the gap size along the azimuthal direction is not limited by the pair production. The pair production can occur at every place in the outer gap, and the emission is assumed to be only outward from the gap. Photon emission outward from an outer gap, projected into the $(\zeta, \Phi)$-plane, is shown in panel a of Figure 7. We give results also for our model: the gap size along the azimuthal direction is limited by the pair production and the pair production occurs only in the region $r_{\text {lim }}-r_{\text {in }}$ of the outer gap. Panel a of Figure 8 shows the photon emission outward from an outer gap (solid curves). The difference in the emission patterns between the RY model and our model is from the limitation of the gap size along the azimuthal direction.

\subsection{Emission inward from the Outer Gap}

In the single-pole outer gap RY model, it was assumed that there is no emission inward from the outer gap to avoid disagreement with observed features of the pulse profile of the Crab, Vela, and Geminga pulsars. However, it is less clear why the physics alone insists that the photons from the outer gap are emitted exclusively outward. In our model, inward emission is from the region $\left(r_{\mathrm{lim}}-r_{\text {in }}\right)$ of the outer gaps. Very high energy emission inward cannot pass freely through the inner magnetosphere because some of these photons are converted into $e^{ \pm}$pairs. When primary photons with energy $E_{\gamma}$ are produced in outer gaps and emitted inward, some will be converted into $e^{ \pm}$pairs on

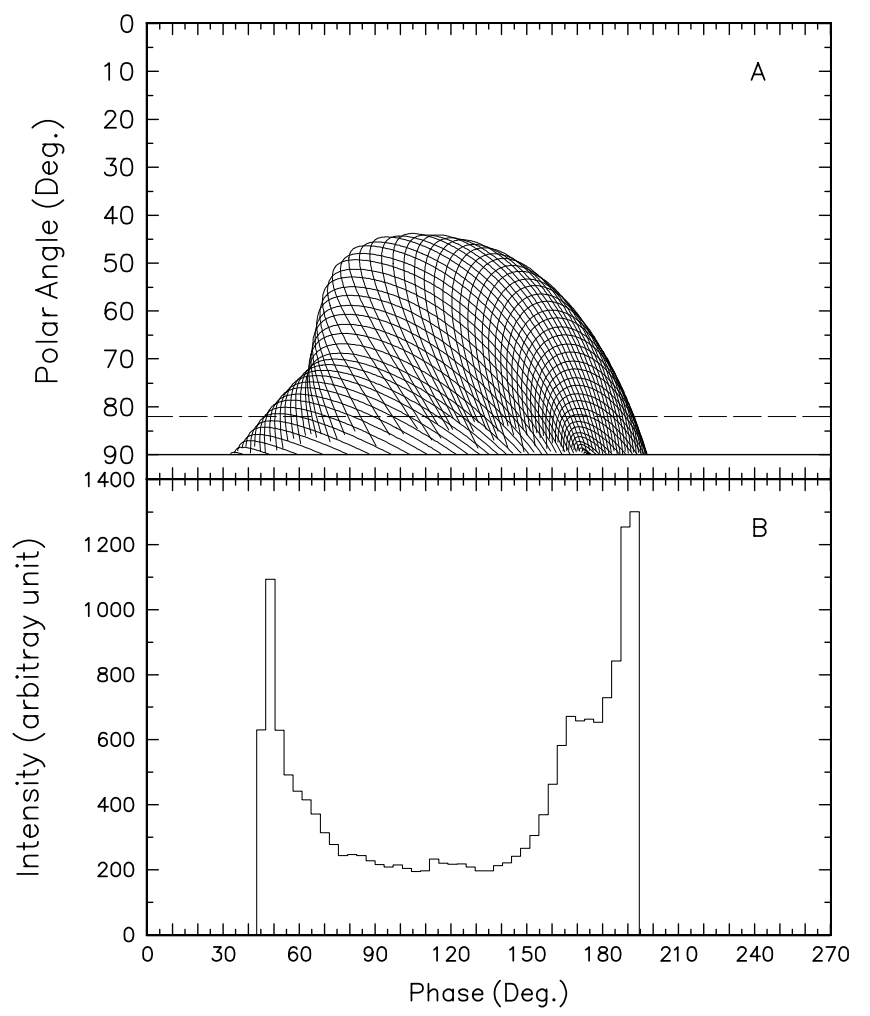

FIG. 7.-Emission projection onto the $(\zeta, \Phi)$-plane and pulse profile for the single-pole outer gap. The photons are emitted outward from the outer gap. (a) The emission projection $\left(a_{1}=0.9\right)$ and $(b)$ the corresponding pulse profile $\left(\Delta a_{1}=0.03\right)$, for Crab parameters $\alpha=65^{\circ}$ and $\zeta=82^{\circ}$.

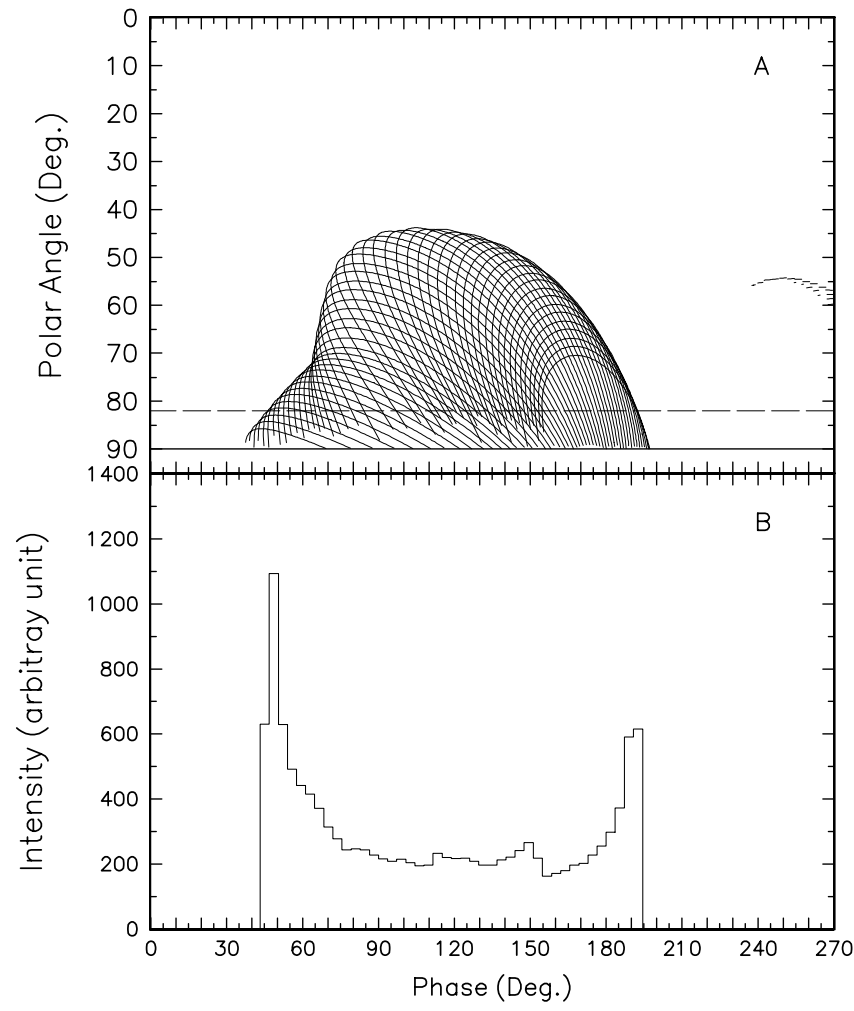

FIG. 8.-Emission projection onto the $(\zeta, \Phi)$-plane and pulse profile. The emission consists of the emission outward from both outer gaps and inward only from the region $\left(r_{\text {lim }}-r_{\text {in }}\right)$ of both outer gaps. The outer gaps are limited along the azimuthal direction by pair production. $(a)$ The emission projection $\left(a_{1}=0.9\right)$ and $(b)$ corresponding pulse profile $\left(\Delta a_{1}=0.03\right)$. Crab parameters, $\alpha=65^{\circ}, \zeta=82^{\circ}$, and $\delta \phi=150^{\circ}$, are used.

both open and closed magnetic field lines in the inner magnetosphere through magnetic pair production. The condition for magnetic pair production is (Sturrock 1971; Ruderman \& Sutherland 1975)

$$
\frac{E_{\gamma}}{2 m_{e} c^{2}} \frac{B\left(r_{s}\right)}{B_{g}} \approx \frac{1}{15},
$$

where $B_{g} \approx 4.4 \times 10^{14} \mathrm{G}$ and $r_{s}$ is the distance to the star from where pairs are produced. From equation (18), the distance $r_{s}$ can be estimated as (ZC; Cheng \& Zhang 1999)

$$
\frac{r_{s}}{R} \approx 0.55 B_{12}^{1 / 3}\left(\frac{E_{\gamma}}{m_{e} c^{2}}\right)^{1 / 3}
$$

Therefore, if $r_{\perp}$ denotes the normal intercept from the center of the star to the tangent to the field line at the point $(x, y, z)$ of the outer gap where the photons are created,

$$
r_{\perp}=\left[r^{2}-\frac{(x \dot{x}+y \dot{y}+z \dot{z})^{2}}{\dot{x}^{2}+\dot{y}^{2}+\dot{z}^{2}}\right]^{1 / 2},
$$

and only photons with $r_{\perp}>r_{s}$ can pass freely through the inner magnetosphere. Photons with $r_{\perp} \leq r_{s}$ will be converted into $e^{ \pm}$pairs. These secondary pairs will emit lower energy photons as synchrotron radiation. A fraction of the synchrotron photons will be further converted into pairs. Moreover, about half of the charged particles accelerated in the outer gap will move inward along the magnetic field lines. These charged particles will produce high-energy photons as curvature radiation. These curvature photons will also be converted into pairs. A cascade will develop 
until the Lorentz factors of the pairs approach unity. Nonthermal X-rays can be produced in this cascade and thermal $\mathrm{X}$-rays can be produced from its impact on the stellar surface (Halpern \& Ruderman 1993; ZC; Cheng et al. 1998; Wang et al. 1998; Cheng \& Zhang 1999). In panel a of Figure 8, that part of the inward emission which can pass freely through the inner magnetosphere is also shown (dashed curves).

\subsection{Pulse Profiles}

We assume that the photon emissivity is uniform along and among outer gap accelerator field lines. For the singlepole outer gap, the pulse-profile corresponding to the emission pattern of panel a of Figure 7 is shown in panel $b$ of Figure 7 in which the viewing angle is assumed to be $80^{\circ}$. It can be seen that the pulse profile is roughly symmetric. In this model, the pulse profile corresponding to the emission pattern of panel a of Figure 8 is shown in panel $b$ of Figure 8. From Figures 7 and 8, it seems that both cases can give roughly the same pulse profile. However, the physics of both models is strikingly different. For the single outer gap model, the emission from the pulsar comes from the emission outward from an outer gap: the emission regions for the two peaks of the pulse profile are those close to the null charge surface and to the light cylinder radius, respectively. However, for the model described above, photon emission consists of emission outward and inward from the region $\left(r_{\text {lim }}-r_{\text {in }}\right)$ of outer gaps limited along the azimuthal direction by $e^{ \pm}$pair production of inward-flowing photons from the outer gaps.

\section{PHASE-RESOLVED SPECTRA OF THE CRAB PULSAR}

Because the Crab pulsar outer gaps are thin, we use the electric field of the CHR model:

$$
E_{||}(r)=\frac{\Omega B(r) a^{2}(r)}{c s(r)}=\frac{\Omega B(r) f^{2}(r) R_{L}^{2}}{c s(r)},
$$

where $a(r)$ is the thickness of the outer gap at position $r$, the radius of the curvature $s \sim\left(r R_{L}\right)^{1 / 2}$, and $f(r) \equiv a(r) / R_{L}$ is the local fractional size of the outer gap. Since the magnetic flux subtended in the outer gap should be constant in the assumed steady state,

$$
f(r) \sim f\left(R_{L}\right)\left(\frac{r}{R_{L}}\right)^{3 / 2},
$$

where $f\left(R_{L}\right)$ is estimated by the pair production condition described in $\S 4$ [n.b., two possible ways to determine $f\left(R_{L}\right)$, eq. (6.7) of CHR II or eq. (22) of ZC]. The local Lorentz factor of the accelerated electrons/positrons in the outer gap is

$$
\gamma_{e}(r)=\left[\frac{3}{2} \frac{s^{2}}{e^{2} c} e E_{||}(r) c\right]^{1 / 4} .
$$

We have used the synchrotron self-Compton model to describe high-energy emission from the Crab pulsar.

In an outer gap, the number of primary charged particles in a volume element $\Delta V$ is roughly given by

$$
d N=n_{\mathrm{GJ}} \Delta A \Delta l,
$$

where $n_{\mathrm{GJ}}=\boldsymbol{\Omega} \cdot \boldsymbol{B} / 2 \pi e c$ is the local Goldreich-Julian number density, $B \Delta A$ is the magnetic flux through the accelerator, and $\Delta l$ is the path length along its magnetic field lines. Using the thin gap approximation, the total number of charged particles in the outer gap is

$$
N \sim \frac{\Omega \Phi}{4 \pi c e} R_{L},
$$

where $\Phi \sim f\left(R_{L}\right) B\left(R_{L}\right) R_{L}^{2} \Delta \phi$ and $\Delta \phi$ is the angular range of the outer gap extending along the azimuthal direction, estimated in $\S 4$. These primary $e^{ \pm}$pairs will lose their energy by radiating curvature photons with a characteristic energy

$$
E_{\mathrm{cur}}(r)=\frac{3}{2} \hbar \gamma_{e}^{3}(r) \frac{c}{s(r)} .
$$

The power into curvature radiation for $d N e^{ \pm}$pairs in a unit volume is

$$
\frac{d L_{\mathrm{cur}}}{d V} \approx l_{\mathrm{cur}} n_{\mathrm{GJ}}(r),
$$

where $l_{\text {cur }}=e E_{||}(r) c$ is the local power into the curvature radiation from a single electron/positron. The spectrum of primary photons from a unit volume is

$$
\frac{d^{2} \dot{N}}{d V d E_{\gamma}} \sim \frac{l_{\text {cur }} n_{\mathrm{GJ}}}{E_{\text {cur }}} \frac{1}{E_{\gamma}}, \quad E_{\gamma} \leq E_{\text {cur }} .
$$

These primary curvature photons collide with the soft photons produced by synchrotron radiation of the secondary $e^{ \pm}$pairs and produce the secondary $e^{ \pm}$pairs by photonphoton pair production. As pointed out in $\S 3.1$, although pair production inside an outer gap is limited to a small region $\left(r_{\text {in }} \leq r \leq r_{\text {lim }}\right)$, pair production outside the outer gap can cover a much wider range because the synchrotron photons produced by the secondary pairs are more abundant than the thermal photons from the stellar surface. The former cannot get into the outer gap because of the field line curvature (cf. CHR I), but they can convert most curvature photons from the outer gap into the secondary pairs. In a steady state, the distribution of secondary electrons/ positrons in a unit volume

$$
\begin{aligned}
\frac{d^{2} N}{d V d E_{e}} & \approx \frac{1}{\dot{E}_{e}} \int \frac{d^{2} \dot{N}\left(E_{\gamma}^{\prime}=2 E_{e}^{\prime}\right)}{d V d E_{\gamma}} d E_{e}^{\prime} \\
& \sim \frac{1}{\dot{E}_{e}} \frac{l_{\text {cur }} n_{\mathrm{GJ}}}{E_{\mathrm{cur}}} \ln \left(\frac{E_{\mathrm{cur}}}{E_{e}}\right),
\end{aligned}
$$

with $\dot{E}_{e}$ the electron energy loss into synchrotron radiation,

$$
\dot{E}_{e}=-\frac{2}{3} \frac{e^{4} B^{2}(r) \sin ^{2} \beta(r)}{m^{2} c^{3}}\left(\frac{E_{e}}{m c^{2}}\right)^{2},
$$

$B(r)$ the local magnetic field and $\beta(r)$ the local pitch angle,

$$
\sin \beta(r) \sim \sin \beta\left(R_{L}\right)\left(\frac{r}{R_{L}}\right)^{1 / 2},
$$

and $\sin \beta\left(R_{L}\right)$ the pitch angle at the light cylinder. Then the energy distribution of the secondary electrons/positrons in volume $\Delta V(r)$,

$$
\begin{aligned}
{\left[\frac{d N(r)}{d E_{e}}\right] } & \approx \frac{d^{2} N}{d V d E_{e}} \Delta V(r) \\
& \sim \frac{1}{\dot{E}_{e}} \frac{l_{\mathrm{cur}} n_{\mathrm{GJ}} \Delta V(r)}{E_{\mathrm{cur}}} \ln \left(\frac{E_{\mathrm{cur}}}{E_{e}}\right) .
\end{aligned}
$$


The corresponding photon spectrum of the synchrotron radiation is

$$
\begin{aligned}
F_{\text {syn }}\left(E_{\gamma}, r\right)= & \frac{3^{1 / 2} e^{3} B(r) \sin \beta}{m c^{2} h} \frac{1}{E_{\gamma}} \\
& \times \int_{E_{\min }}^{E_{\max }}\left[\frac{d N(r)}{d E_{e}}\right] F(x) d E_{e},
\end{aligned}
$$

where $x=E_{\gamma} / E_{\text {syn }}$. The typical photon energy is

$$
E_{\mathrm{syn}}(r)=\frac{3}{2}\left(\frac{E_{e}}{m c^{2}}\right)^{2} \frac{h e B(r) \sin \beta(r)}{m c},
$$

and $F(x)=x \int_{x}^{\infty} K_{5 / 3}(y) d y$, where $K_{5 / 3}(y)$ is the modified Bessel function of order $5 / 3$. Similarly, the spectrum of inverse Compton scattered photons in the volume $\Delta V(r)$ is

$$
F_{\mathrm{ICS}}\left(E_{\gamma}, r\right)=\int_{E_{\min }}^{E_{\max }}\left[\frac{d N(r)}{d E_{e}}\right]\left[\frac{d^{2} N_{\mathrm{ICS}}(r)}{d E_{\gamma} d t}\right] d E_{e},
$$

where

$$
\begin{gathered}
\frac{d^{2} N(r)_{\mathrm{ICS}}}{d E_{\gamma} d t}=\int_{\epsilon_{1}}^{\epsilon_{2}} n_{\mathrm{syn}}(\epsilon, r) F\left(\epsilon, E_{\gamma}, E_{e}\right) d \epsilon, \\
F\left(\epsilon, E_{\gamma}, E_{e}\right)=\frac{3 \sigma_{T} c}{4\left(E_{e} / m c^{2}\right)^{2}} \frac{1}{\epsilon}[2 q \ln q+(1+2 q)(1-q) \\
\left.+\frac{(\Gamma q)^{2}(1-q)}{2(1+\Gamma q)}\right],
\end{gathered}
$$

with $\Gamma=4 \epsilon\left(E_{e} / m c^{2}\right) / m c^{2}, q=E_{1} / \Gamma\left(1-E_{1}\right)$ with $E_{1}=$ $E_{\gamma} / E_{e}$, and $\frac{1}{4}\left(E_{e} / m c^{2}\right)<q<1$. The number density of the synchrotron photons with energy $\epsilon$ is

$$
n_{\mathrm{syn}}(\epsilon, r)=\frac{F_{\mathrm{syn}}(\epsilon)}{c r^{2} \Delta \Omega},
$$

where $F_{\text {syn }}$ is the calculated synchrotron radiation flux and $\Delta \Omega$ is the usual beam solid angle.

Despite the fact that the outer gap has a finite thickness, we have used only one representative, $a_{1}=0.9$. For a thin outer gap, this seems a reasonable approximation. Then we calculate the radial distances which correspond to various pulse phases for a given $a_{1}$. We assumed that high-energy photons are emitted tangentially to the local magnetic field. For given viewing angles to the Crab pulsar and a constant $a_{1}$ surface, the emission regions as functions of $\phi$ and $r$ on the $a_{1}$ surface is shown in Figure 9. Once the radial distances are determined, the spectrum of photon emission can be calculated for a given radial distance $r$. In order to compare with the observed phase-resolved spectra of Crab, we divide the calculated pulse phases into different parts according to the division given for the observed phases; each part determines the possible values of the radial distances.

We have calculated the phase-resolved spectra for the phases labeled peak 1 , trailing wing 1 , bridge, leading wing 2 , and peak 2 . These divisions are those given by Fierro et al. (1998). In our calculations, $a_{1} \sim 0.9, f\left(R_{L}\right) \sim 0.21$, and $\sin \beta\left(R_{L}\right) \sim 0.085$ are used. The comparisons of model results with the observed phase-resolved spectra of Crab are shown in Figure 10, in which the amplitude of the calculated spectra have been adjusted according to Figure 8. It can be seen that our model results are consistent with the observations.

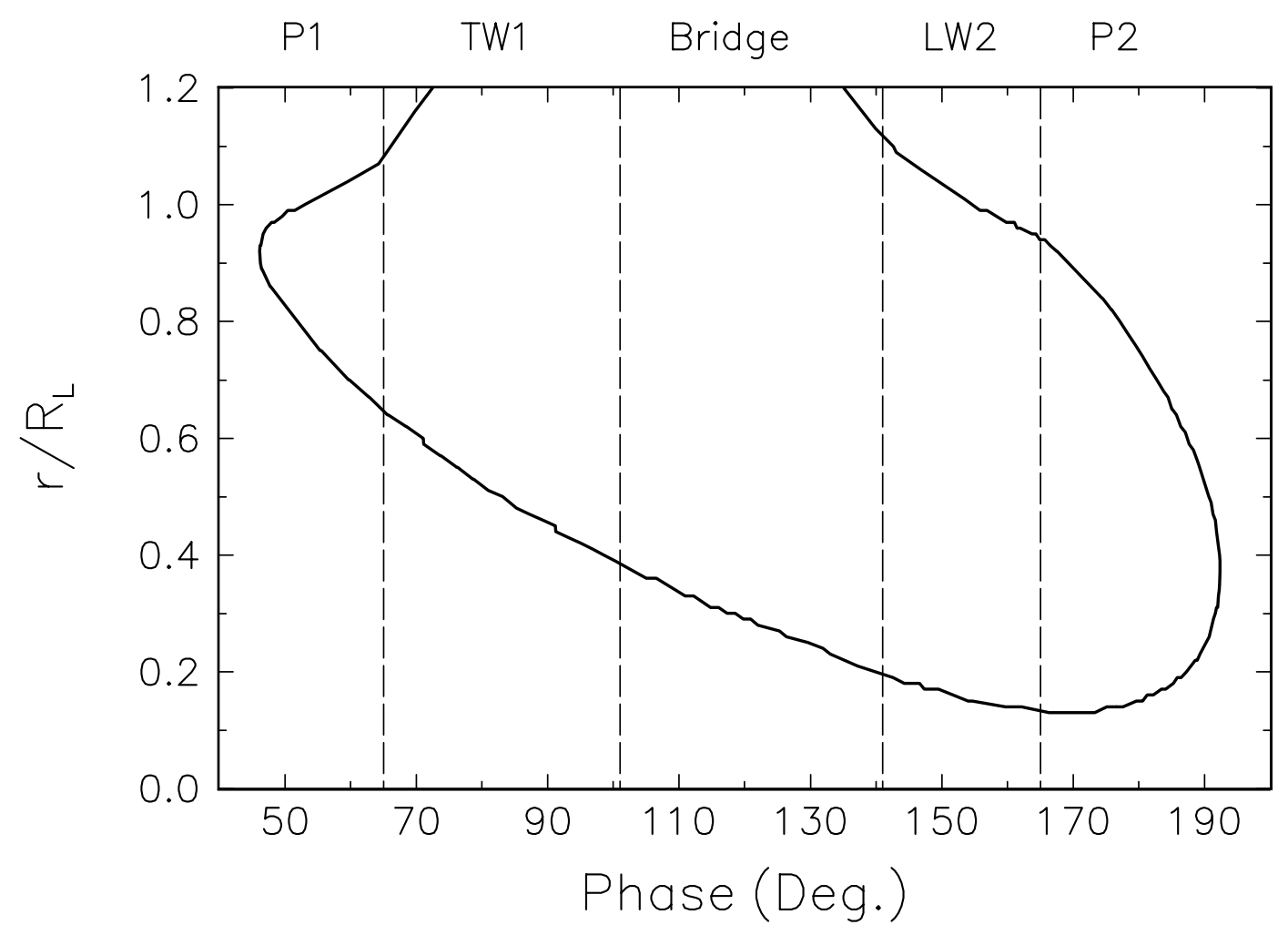

FIG. 9.-Variation of radial distance with pulse phase for the Crab. The inclination angle is $65^{\circ}$ and $a_{1}=0.9$. Five regions for different pulse phase are indicated. 

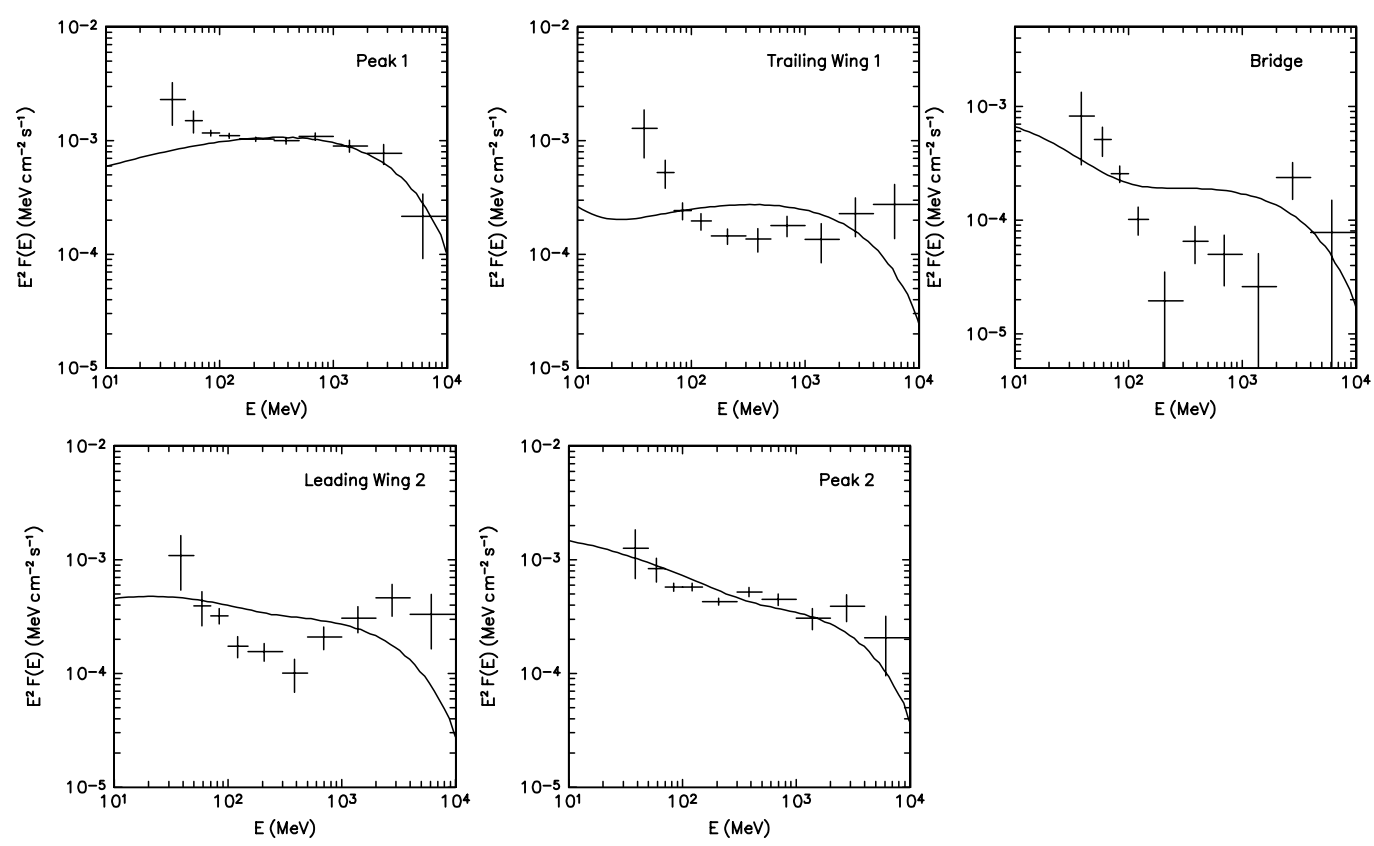

Fig. 10.- Phase-resolved $\gamma$-ray spectra for the different phases of the Crab pulsar: peak 1, trailing wing 1, bridge, leading wing 2, and peak 2. Observed data are taken from Fierro et al. (1998).

The calculated phase-averaged spectrum is shown in Figure 11.

\section{SUMMARY AND DISCUSSION}

We have reconsidered the geometry of outer gap accelerators in the three-dimensional magnetospheric pulsar model, following groundbreaking work by Romani and his coworkers (RY; Yadigaroglu \& Romani 1995). In our model, however, the region of the photon-photon pair production in the outer gaps and the limit of the photonphoton pair production on the extension of the outer gaps along the azimuthal direction have been explicitly taken into account. We find that the two longer outer gaps (gap 1 and gap 2), with some extension in the azimuthal $(\phi)$ direction, exist in the pulsar magnetosphere. We have also considered the effect of the magnetic pair production in the inner magnetosphere and find that only photons with $r_{\perp}>$ $r_{s}$ produced in the outer gaps should pass freely through the inner magnetosphere. Therefore, in our model doublepeaked pulse profiles with varying phase separation, which depend on the viewing angle, and strong bridge emission occur naturally, as in the single-pole outer gap model. However, we also have more emission morphology possibilities than those with a single-pole outer gap. We have

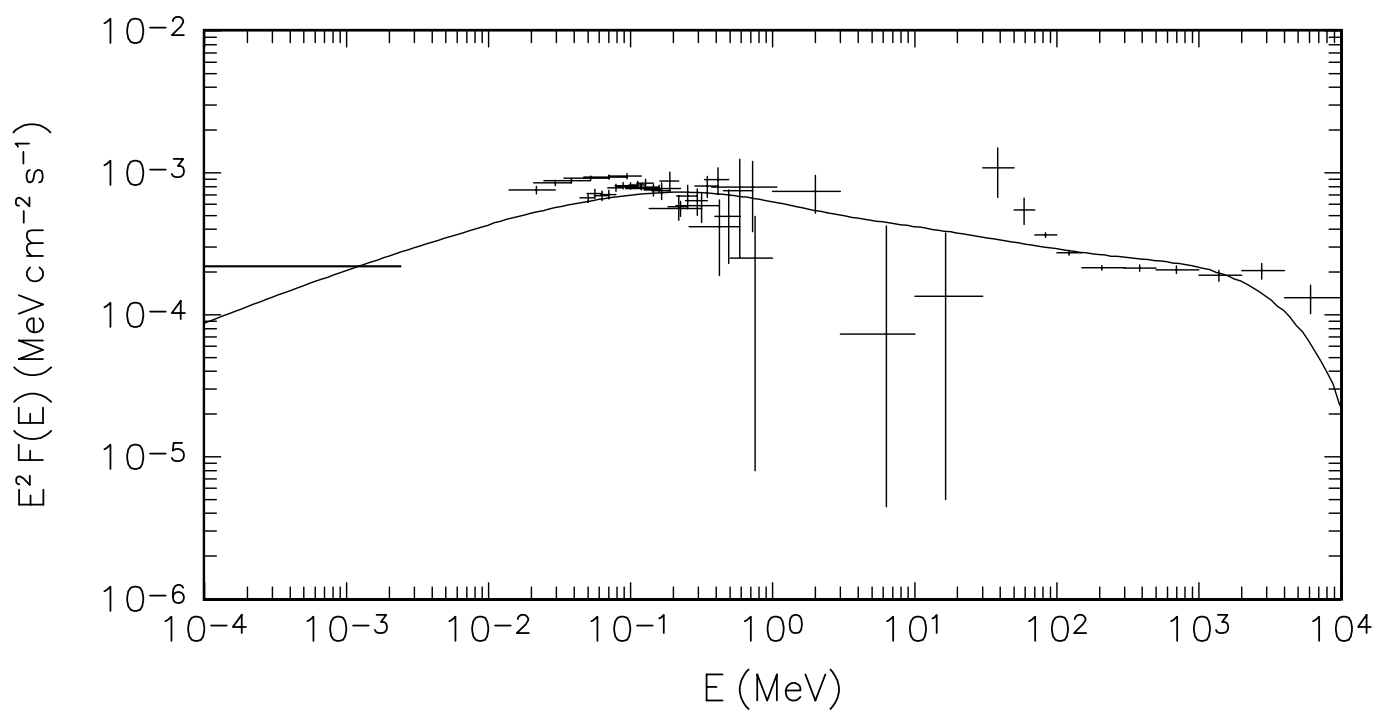

FIG. 11.-Phase-averaged spectrum for the Crab. The observed ROSAT data is from Becker \& Trümper (1997). The observed OSSE, BATSE, and COMPTEL data are taken from Ulmer et al. (1995). The EGRET data are from Fierro et al. (1998). 
applied our model to the Crab pulsar's phase-resolved spectra, and results consistent with the observation are obtained.

In this model, the pulse profile is mainly determined by emission outward from an outer gap, although emission inward along the magnetic field lines has been taken into account. The observed consequences are essentially just those already predicted by RY. The emission pattern and pulse profile depend on the magnetic inclination angle. Observed pulse separation depends on both the magnetic inclination angle and the viewing angle. It increases with decreasing magnetic inclination for a given viewing angle. The magnetic inclination angles of the $\gamma$-ray pulsars with large pulse separations such as Geminga and PSR B1951 + 32 should be smaller. Observed pulse profiles (Fierro et al. 1998) in different energy ranges for Crab, Vela, and Geminga have been obtained. A common feature is that nonzero off-pulse emission decreases rapidly with energy, but peak and bridge emissions do not. Lower energy photons dominate the nonzero off-pulse emission. In our model, the off-pulse emission near the first peak is produced by the cascade process in the inner magnetosphere: photons emitted inward and charged particles which move inward along the magnetic field lines (from the same outer gap) are responsible for this cascade process. The off-pulse emission near the second peak is produced by this same cascade process, but the photons and charged particles responsible for this process come from the opposite outer gap.

In fitting the phase-resolved spectra of the Crab pulsar, our model can fit the observed data roughly. From Figure
10 , we see that the observed intensity data are higher than those of model calculation in low-energy parts of P1, TW1, Bridge, and LW2, which implies that the synchrotron component is stronger than the model calculations. In the highenergy parts of the phase-resolved spectra, the data are also higher than the model results. We speculate that some of the primary curvature photons escape conversion by the secondary X-rays. Therefore, a curvature component with a typical energy $\sim 10 \mathrm{GeV}$ shows up in the high-energy tail of the observed phase-resolved spectra. In our model calculation, only one surface layer of the magnetic field lines is used and photons are assumed to be emitted tangent to the local field lines. Therefore, we cannot produce leading-edge and trailing-edge emission. However, some of the secondary pairs have quite large initial pitch angles whose emission will not be tangent to the local field lines. In future calculations, these should be taken into account. Finally, although our calculation is based on a steady state accelerator, a realistic outer magnetospheric gap may be a dynamical or flickering one.

We thank an excellent anonymous referee; R. W. Romani, J. Gil, and W. Becker for their constructive suggestions and discussion; P. L. Nolan and J. M. Fierro for providing us with EGRET data from the Crab pulsar; and M. P. Ulmer for OSSE, BATSE, and COMPTEL data. This work is partially supported by an RGC grant of the Hong Kong Government and a Croucher Foundation Senior Research Fellowship.

\section{APPENDIX A}

\section{THE STATIC DIPOLE FIELD}

Using equation (1), the three components of the static dipole field in Cartesian coordinates are

$$
\begin{aligned}
& B_{x}^{\text {sd }}=\frac{\mu}{r^{5}}\left[3 x z \cos \alpha+\left(3 x^{2}-r^{2}\right) \sin \alpha\right], \\
& B_{y}^{\text {sd }}=\frac{\mu}{r^{5}}(3 y z \cos \alpha+3 x y \sin \alpha), \\
& B_{z}^{\text {sd }}=\frac{\mu}{r^{5}}\left[\left(3 z^{2}-r^{2}\right) \cos \alpha+3 x z \sin \alpha\right] .
\end{aligned}
$$

It has been assumed above that the rotation axis is directed along the $z$-axis, $\mu$ lies in the $(x, z)$-plane, and the angle between the magnetic axis and the rotation axis is $\alpha$. From the above equations, the three-dimensional structure of the magnetosphere is determined. In a static dipole field, several characteristic quantities which describe the basic feature of the magnetosphere structure are as follows. First, the opening angle of the last closed field line (i.e., the angle between $\mu$ and $\boldsymbol{B}$ ) in spherical coordinates is

$$
\cos \omega=Z-2 Z^{-1}
$$

where

$$
Z \equiv\left(3 \sin ^{2} \theta \cos ^{2} \phi \sin ^{2} \alpha+6 \sin \theta \cos \theta \cos \phi \sin \alpha \cos \alpha+3 \cos ^{2} \theta \cos ^{2} \alpha+1\right)^{1 / 2} .
$$

Second, the polar angle which defines the null surface $(\boldsymbol{\Omega} \cdot \boldsymbol{B}=0)$ is given by

$$
\cos ^{2} \theta_{\text {null }}=\frac{1}{2}-\frac{1 / 3 \pm \chi_{1}\left(\chi_{1}^{2}+8 / 9\right)^{1 / 2}}{2\left(1+\chi_{1}^{2}\right)}
$$


with

$$
\chi_{1} \equiv \tan \alpha \cos \phi .
$$

The null surface can be obtained from a series of values of $\phi$ from 0 to $2 \pi$ for a given $\alpha$. We can determine the polar angle at which a field line is tangential to the light cylinder from

$$
\sin ^{2} \theta_{r l}=\frac{1}{3}+\frac{1 \pm 3\left(1+8 \chi_{2}^{2}\right)^{1 / 2}}{6\left(1+9 \chi_{2}^{2}\right)}
$$

with

$$
\chi_{2} \equiv \frac{1}{3} \tan \alpha \cos \phi
$$

\section{APPENDIX B}

\section{THE ROTATING DIPOLE FIELD}

Using equations (2) and (3), the magnetic field in Cartesian coordinates,

$$
\boldsymbol{B}=B_{x} \hat{\boldsymbol{x}}+B_{y} \hat{\boldsymbol{y}}+B_{z} \hat{z},
$$

is

$$
\begin{aligned}
B_{x}= & \frac{\mu}{r^{5}}\left[\left[3 x z \cos \alpha+\sin \alpha\left\{\left[\left(3 x^{2}-r^{2}\right)+3 x y\left(r / R_{L}\right)+\left(r^{2}-x^{2}\right)\left(r / R_{L}\right)^{2}\right]\right.\right.\right. \\
& \left.\left.\left.\times \cos \left[(R-r) / R_{L}\right]+\left[3 x y-\left(3 x^{2}-r^{2}\right)\left(r / R_{L}\right)-x y\left(r / R_{L}\right)^{2}\right] \sin \left[(R-r) / R_{L}\right]\right\}\right]\right], \\
B_{y}= & \frac{\mu}{r^{5}}\left[\left[3 y z \cos \alpha+\sin \alpha\left\{\left[3 x y+\left(3 y^{2}-r^{2}\right)\left(r / R_{L}\right)-x y\left(r / R_{L}\right)^{2}\right]\right.\right.\right. \\
& \left.\left.\left.\times \cos \left[(R-r) / R_{L}\right]+\left[\left(3 y^{2}-r^{2}\right)-3 x y\left(r / R_{L}\right)+\left(r^{2}-y^{2}\right)\left(r / R_{L}\right)^{2}\right] \sin \left[(R-r) / R_{L}\right]\right\}\right]\right], \\
B_{z}= & \frac{\mu}{r^{5}}\left[\left[\left(3 z^{2}-r^{2}\right) \cos \alpha+\sin \alpha\left\{\left[3 x z+3 y z\left(r / R_{L}\right)-x z(r / R L)^{2}\right]\right.\right.\right. \\
& \left.\left.\left.\times \cos \left[(R-r) / R_{L}\right]+\left[3 y z-3 x z\left(r / R_{L}\right)-y z\left(r / R_{L}\right)^{2}\right] \sin \left[(R-r) / R_{L}\right]\right\}\right]\right] .
\end{aligned}
$$

In the above derivations, the retarded time $t_{r}=(R-r) / c$ is used, with $\Omega t_{r}=(R-r) / R_{L}$, where $R$ is the radius of the neutron star, $r$ is the radial distance to the star, and $R_{L}$ is the radius of the light cylinder.

\section{REFERENCES}

Arendt, P. N., \& Eilek, J. A. 1998, ApJ, submitted (preprint astro-ph/ 9801257)

Becker, W., \& Trümper, J. 1997, A\&A, 326, 682

Biggs, J. D. 1990, MNRAS, 245, 514

Cheng, A. F., Ruderman, M. A., \& Sutherland, P. G. 1976, ApJ, 203, 209

Cheng, K. S., Gil, J., \& Zhang, L. 1998, ApJ, 493, L35

Cheng, K. S., Ho, C., \& Ruderman, M. A. 1986a, ApJ, 300, 500 (CHR I) . 1986b, ApJ, 300, 522 (CHR II)

Cheng, K. S., \& Zhang, L. 1999, ApJ, 515, 337

Chiang, J., \& Romani, R. W. 1992, ApJ, 400, 629 1994, ApJ, 436, 754

Fierro, J. M. 1995, Ph.D. thesis, Stanford Univ.

Fierro, J. M., Michelson, M., Nolan, P. L., \& Thomson, D. J. 1998, ApJ, 494, 734

Goldreich, P., \& Julian, W. H. 1969, ApJ, 157, 869

Halpern, J. P., \& Ruderman, M. A. 1993, ApJ, 415, 286

Ho, C. 1986, MNRAS, 221, 523

. 1989, ApJ, 342, 396

Holloway, N. J. 1973, Nature Phys. Sci., 246, 6

Jackson, J. D. 1975, Classical Electrodynamics (New York: Wiley)
Roberts, D. H., \& Sturrock, P. A. 1972, ApJ, 173, L33

Romani, R. W. 1996, ApJ, 470, 469

Romani, R. W., \& Yadigaroglu, I.-A. 1995, ApJ, 438, 314 (RY)

Ruderman, M. A. 1991a, ApJ, 366, 261

-. 1991b, ApJ, 382, 576 .1991c, ApJ, 382, 587

2000, in IAU Symp. 195, Highly Energetic Physical Processes and Mechanisms for Emission from Astrophysical Plasmas, ed. P. C. H. Martens, S. Tsuruta, \& M. A. Weber (San Francisco: ASP), 463

Ruderman, M. A., \& Sutherland, P. G. 1975, ApJ, 196, 51

Sturrock, P. A. 1971, ApJ, 164, 529

Thompson, D. J., et al. 1996, ApJ, 465, 385

Ulmer, M. P., et al. 1995, ApJ, 448, 356

Wang, F. Y.-H., Ruderman, M., Halpern, J. P., \& Zhu, T. 1998, ApJ, 498, 373

Yadigaroglu, I.-A. 1997, Ph.D. thesis, Stanford Univ.

Yadigaroglu, I.-A., \& Romani, R. W. 1995, ApJ, 449, 211

Zhang, L., \& Cheng, K. S. 1997, ApJ, 487, 370 (ZC)

Zhu, T., \& Ruderman, M. A. 1997, ApJ, 478, 701 